\title{
Economic Assessment of the Relationship between Disease Exacerbations and the Cost of Multiple Sclerosis
}

OBJECTIVE: The primary purpose of this study was to define the cost of multiple sclerosis (MS) from the perspective of a managed care organization (MCO). This study also estimated the cost of a disease exacerbation to predict the cost that may be offset by the use of disease-modifying pharmaceutical agents.

DESIGN: Retrospective (1995-96) claims of a managed care medical and pharmacy database were used as data sources. Multiple linear regression was used to identify the determinants of cost and to estimate the cost of treating an MS disease exacerbation.

SETTING: A group model HMO covering approximately 325,000 lives.

PATIENTS: Patients continuously enrolled during the study period with a claim for MS (ICD-9-340) or demyelinating disease (ICD-9-341) were included in the study.

MAIN OUTCOME MEASURES: Total cost, cost per exacerbation episode, determinants of cost

RESULTS: The total cost to the MCO for the care of MS patients averaged $\$ 9,365(+/-\$ 11,047)$ per patient for the two-year study period. Principal determinants of cost were the number of exacerbations, comorbidities, and inter-feron beta claims, cardiovascular comorbidities specifically identified as a significant predictor of cost. The average cost of a disease exacerbation differed depending on the number of comorbidities the patient had, ranging from $\$ 359$ for a patient with no comorbidities to $\$ 2,839$ for a patient with eight.

CONCLUSION: Managed care decision makers may wish to target MS patients with multiple comorbidities for disease-modifying therapy, because the cost of treating an exacerbation was found to be higher for these patients. This study provides a methodological framework that may be used to assess the potential impact of a disease-modifying agent on a managed care budget.

KEYWORDS: Cost, economic, interferon, multiple sclerosis

J Managed Care Pharm 2000: 19-24

\section{Authors}

AMY NICOLE GRUDZINSKI, Pharm.D., M.S., is Director, Outcomes Research, Pfizer Inc., Ncw York, NY. At the time this study was conducted, she was affiliated with the University of Arizona, Tucson, $A Z$, and Roche Global Pharmacocconomic Research, Palo Alto, CA; ZAFAR HAKIM, Ph.D., is Manager, Decision Analysis, Roche Global Pharmacoeconomic Research; EMILY R. COX, Ph.D., is Assistant Professor at the University of Arizona; DAVID M. LABINER, M.D., is Associate Professor at the University of Arizona, and J. LYLE BOOTMAN, Ph.D., is Dean and Professor, the Universily of Arizona.

ACKNOWLEDGEMENT: The authors would like to thank Amy J. Jones, Pharm. D., and Louis P. Garrison, Ph.D., of Roche Pharmaceuticals for their contribution to the study design. Funding for this study was provided through an uncstricted educational fellowship grant from Roche Pharmaceuticals.

AUTHOR CORRESPONDENCE: Amy Nicole Grudzinski, Pharm.D., M.S., Director, Outcomes Research, Pfizer Inc., 235 East 42nd Street, New York, NY 10017-5755. E-mail: grudza@pfizercom.

Copyrighı@ 2000 Academy of Managed Care Pharmacy. Inc. All rights reserved.

\author{
by Amy Nicole Grudzinski, Zafar Hakim, \\ Emily R. Cox, David M. Labiner, and \\ J. Lyle Bootman
}

1 ultiple sclerosis (MS) is an incurable, neurodegenerative disease that affects approximately 95 persons in $100,000,1,2$ To maintain functioning and alleviate symptoms, patients must undergo lifelong treatment. The introduction of expensive disease-modifying pharmaceutical agents that may decrease the number of MS exacerbations may increase the cost of treating these patients

The majority of insured American workers are now covered under managed care plans. ${ }^{3}$ In 1997 managed care dollars represented approximately half of all drug reimbursement in the U.S. ${ }^{4}$ Yet insufficient pharmacoeconomic information exists that specifically caters to the needs of managed care decision makers concerned with the direct cost of treating representative patient populations under real-world conditions. ${ }^{5}$ Economic studies that report cost by MS disease severity level have been published. ${ }^{6,7}$ However, the patient samples and costs included in these studies may differ substantially from those found in the managed care environment. The estimates derived from these studies may not be applicable to a managed care population.

The objectives of this study were to define the cost of treating MS from the perspective of the managed care organization (MCO), to identify the determinants of this cost, and to estimate the cost of treating an MS disease exacerbation. To accomplish these objectives, a cost-of-illness analysis was conducted using a managed care claims database. Multiple linear regression analysis was used to identify the principal determinants of cost from a range of patient-and treatment-related factors. Following the identification of the principal determinants of cost, inferences were drawn with respect to the impact that a change in one or more of these variables might have on the total cost to the MCO. The relationship between disease severity and cost was examined to assess the potential impact of a disease-modifying agent on a managed care budget.

\section{Method}

Data were obtained from a group-model $\mathrm{HMO}$ in Arizona covering approximately 325,000 lives. Coverage was fairly comprehensive and included physician office visits, specialist visits, lab tests, urgent care, hospitalization, physical therapy, preventative care, immunizations, allergy testing, vision and hearing exams, family planning, and prescriptions. 
Patients continuously enrolled from January 1,1995 , through December 31, 1996, with a primary or secondary claim for MS (ICD-9-340) or demyelinating disease (ICD-9-341) were included in the study. Patients were excluded if coverage commenced or terminated or if lapses in coverage exceeding 45 days occurred during the study period. The 45-day period was chosen to define a lapse in coverage to avoid excluding patients on the basis of temporary monthly errors in data collection processing.

\section{Disease Severity}

Disease severity was based on the number of disease exacerbations a patient experienced during the study period. Disease exacerbations may be defined as periods of clinical worsening after which patients may return to baseline disability levels or maintain a residual deficit. ${ }^{8}$ The following criteria, based on typical treatment of an exacerbation, were used to define the number of exacerbations a patient experienced during the study period: (1) a hospital claim listing ICD-9-340 or 341 as the primary or secondary diagnosis; (2) an emergency room claim listing ICD-9-340 or 341 as the primary or secondary diagnosis; or (3) a pharmacy claim for an oral or intravenous steroid. The total number of exacerbations a patient experienced could be any combination of emergency room (ER) or hospital claims listing ICD-9-340 or 341, or prescription steroid claims. A 42day block-out period was observed after each exacerbation, during which patients were not eligible to be considered as having another exacerbation. For example, if an ER claim was filed for a patient listing an ICD-9 of 340 or 341 , and a hospital claim listing ICD-9-340 or 341 was filed the following day, the patient would be considered to have only one exacerbation episode. This time period was chosen on the basis of documented literature that indicates MS disease exacerbations last approximately six weeks. ${ }^{8}$

\section{Cost}

Cost was identified using medical and pharmacy claims data for the study sample. Given the perspective of the analysis, indirect and intangible costs were not estimated. A prevalence-based approach was used to estimate the cost to an $\mathrm{MCO}$ of treating patients with MS, irrespective of the time since onset. For descriptive purposes, total costs were broken down into two all-inclusive categories: pharmacy costs and medical costs. Each of these categories was further divided into MS-related and nonMS-related costs. MS-related pharmacy cosis were derived by reviewing all pharmacy claims for the study sample and including only those for pharmaceuticals indicated for the primary treatment of MS. The amount of MS-related medical cost was compiled based on the cost of all claims that listed an ICD9 of 340 or 341 as a primary or secondary diagnosis.

\section{Regression Analysis}

Ordinary least squares regression was used to identify the principal determinants of the cost of care for MS. Diagnostic tests were performed to ensure that the data met the basic assumptions of the linear regression model. The data were analyzed using both total costs and the log of total costs as the dependent variable. The untransformed version of the dependent variable was selected for presentation because the data better met the assumptions of linear regression in this form.

The dependent variable in all regressions was total direct medical and pharmacy costs for services covered by the MCO. Independent variables included the patient's age, gender, interferon beta claims, comorbidities, and exacerbations. The number of disease exacerbations experienced by the patient was used as a proxy for disease severity. All other independent variables were included in the regression model as controls to account for patient characteristics that may have affected variations in costs to the MCO. All independent variables entered the model as continuous variables, with the exception of binary variables indicating patient's gender and the presence of specific (mental, cardiovascular, genitourinary) comorbidities.

Patient's age was selected as a control variable to account for age-related variations in costs. Because the effects of MS tend to increase with years since diagnosis, older patients were expected to have more severe disease than younger patients in general. Information on the patients' years since disease onset was not available in the database used; however, increasing MS health care resource utilization rates with increasing age demonstrated in a previous study suggested that this variable should be included in this analysis. ${ }^{6}$

Patients' gender was used as a control variable on the basis of the documented differences that exist in regard to disease course, disease severity, and resource utilization associated with the treatment of MS in males and females. ${ }^{9.10}$ The number of interferon beta claims was chosen as an independent variable due to its high acquisition cost, which was likely to explain some of the variation in total health care costs.

Patients' comorbidities were expected to affect total costs as well as the costs associated with MS treatment, because these patients may require hospitalization and/or additional monitoring during their MS treatment. A continuous variable that indicated the number of comorbidities by ICD-9-CM category (1994, Hospital edition, Volume 1) was used to account for the impact of comorbidities on resource utilization. Patients with hypertension in particular were recognized as likely candidates for additional monitoring, medications, and hospitalization during corticosteroid treatment for an MS exacerbation. Thus, the presence of cardiovascular comorbidities stood to substantially affect the cost of treating MS in these patients. A previous study had shown that MS patients with genitourinary complications generated higher than average medical costs.? Additionally, the high incidence of suicide associated with MS ${ }^{11}$ and the substantial expense of treating depression ${ }^{12}$ suggested mental comorbidities should also be examined in this analysis. For these reasons, specific binary variables indicating the presence of cardiovascular, genitourinary, and mental comorbidities were created. Separate regressions were estimated using either the continuous variable indicating the number of all comorbidities or the three binary variables to examine the effect of comorbidities on total cost. 
The regression models estimated are: TOTCOST indicates total health care costs, pharmacy plus medical; AGE indicates the age at beginning of study period in years; GENDER indicates $0=$ male or 1 =female patient; MENTAL, CARDIO, and GENITO indicate the $0=$ absence or 1 =presence of mental, cardiovascular, or genitourinary comorbidities, respectively; IBCLMS indicates the number of interferon beta prescription claims; COMGRP indicates the number of comorbid disease state groups; and EXCER indicates the number of exacerbations as previously defined.
Equation 1: TOTCOST $=\beta_{0}+\beta_{1} E X C E R+\beta_{2} A G E+\beta_{3} G E N D E R$

$+\beta 4 M E N T A L+\beta_{5} C A R D I O+\beta_{6} G E N I T O+\beta_{7} I B C L M S+\varepsilon_{1}$

Equation 2: TOTCOST $=\beta_{0}+\beta_{1}$ EXCER $+\beta_{2}$ AGE $+\beta_{3}$ GENDER $+\beta+C O M G R P+\beta 3 I B C L M S+\equiv \mathrm{E}_{1}$

Two sets of regression equations were estimated. The first set (including equation 1) treated comorbidities as a categorical variable and the second set (including equation 2) treated comorbidities as a continuous variable. Within each set of regressions two different regression models were estimated. The first

\section{NO EXACERBATIONS}

\begin{tabular}{|c|c|c|c|c|c|c|}
\hline \multirow[t]{2}{*}{ Variable } & \multirow[t]{2}{*}{$\mathbf{N}$} & \multirow[t]{2}{*}{ Mean } & \multirow{2}{*}{$\begin{array}{l}\text { Standard } \\
\text { deviation }\end{array}$} & \multirow[t]{2}{*}{ Median } & \multicolumn{2}{|c|}{ Interquartile Range } \\
\hline & & & & & Q1 & Q3 \\
\hline Total cost & 1.56 & $\$ 6007$ & $\$ 6742$ & $\$ 3299$ & $\$ 1006$ & $\$ 7821$ \\
\hline Pharmacy cost & 156 & $\$ 3001$ & $\$ 4277$ & $\$ 736$ & $\$ 138$ & $\$ 3667$ \\
\hline MS-related pharmacy cost & 156 & $\$ 2592$ & $\$ 4183$ & $\$ 170$ & $\$ 83$ & $\$ 3039$ \\
\hline Medical cost & 156 & $\$ 3006$ & $\$ 3409$ & $\$ 1623$ & $\$ 504$ & $\$ 5108$ \\
\hline MS-related medical cost & 156 & $\$ 537$ & $\$ 1034$ & $\$ 174$ & $\$ 61$ & $\$ 556$ \\
\hline
\end{tabular}

\section{ONE EXACERBATION}

\begin{tabular}{|c|c|c|c|c|c|c|}
\hline Total cost & 89 & $\$ 8180$ & $\$ 8122$ & $\$ 5556$ & $\$ 2730$ & $\$ 10,768$ \\
\hline Pharmacy cost & 89 & $\$ 2678$ & $\$ 3694$ & $\$ 1034$ & $\$ 257$ & $\$ 3521$ \\
\hline MS-related pharmacy cost & 89 & $\$ 2144$ & $\$ 3611$ & $\$ 485$ & $\$ 42$ & $\$ 2214$ \\
\hline Medical cost & 89 & $\$ 5502$ & $\$ 6231$ & $\$ 3912$ & $\$ 1639$ & $\$ 7224$ \\
\hline MS-related medical cost & 89 & $\$ 1004$ & $\$ 1954$ & $\$ 381$ & $\$ 100$ & $\$ 1085$ \\
\hline
\end{tabular}

\section{TWO EXACERBATIONS}

\begin{tabular}{l|c|c|c|c|c}
\hline Total cost & 42 & $\$ 14,521$ & $\$ 13,830$ & $\$ 11,792$ & $\$ 5049$ \\
\hline Pharmacy cost & 42 & $\$ 2664$ & $\$ 3989$ & $\$ 1364$ & $\$ 343$ \\
\hline MS-related pharmacy cost & 42 & $\$ 2111$ & $\$ 3636$ & $\$ 469$ & $\$ 2315$ \\
\hline Medical cost & 42 & $\$ 11,857$ & $\$ 13,324$ & $\$ 7,468$ & $\$ 2,805$ \\
\hline MS-related medical cost & 42 & $\$ 4812$ & $\$ 6986$ & $\$ 1383$ & $\$ 367$ \\
\hline
\end{tabular}

THREE TO EIGHT EXACERBATIONS

\begin{tabular}{l|c|c|c|c|c|c}
\hline Total cost & 37 & $\$ 20,519$ & $\$ 17,579$ & $\$ 17,898$ & $\$ 9147$ \\
\hline Pharmacy cost & 37 & $\$ 3081$ & $\$ 3293$ & $\$ 2162$ & $\$ 487$ \\
\hline MS-related pharmacy cost & 37 & $\$ 1328$ & $\$ 2509$ & $\$ 356$ & $\$ 4063$ \\
\hline Medical cost & 37 & $\$ 17,437$ & $\$ 15,666$ & $\$ 14,603$ & $\$ 7531$ & $\$ 1393$ \\
\hline MS-related medical cost & 37 & $\$ 4735$ & $\$ 7865$ & $\$ 912$ & $\$ 158$ & $\$ 6891$ \\
\hline
\end{tabular}




\begin{tabular}{|c|c|c|c|c|c|c|}
\hline \multirow[b]{3}{*}{ VARIABLES } & \multicolumn{6}{|c|}{$\begin{array}{l}\text { Linear Regression Parameter Estimates ( } \beta \text { ) and Standard Errors (SE) Using the Number of } \\
\text { Disease Exacerbations as the Indicator of Disease Severity; Dependent Variable=Total Costs } \\
1995-96(n=324)\end{array}$} \\
\hline & \multicolumn{2}{|c|}{ EQUATION 1} & \multicolumn{2}{|c|}{ EQUATION 2} & \multicolumn{2}{|c|}{ EQUATION 3} \\
\hline & Beta & SE & Beta & SE & Beta & SE \\
\hline Intercept & 960 & 1860 & -2110 & 1734 & -468 & 1110 \\
\hline Exacerbations ${ }^{1}$ & $3829 * * *$ & 385 & $2811^{* * *}$ & 386 & 359 & 820 \\
\hline $\mathrm{Age}^{2}$ & 13 & 40 & -9 & 32 & & \\
\hline Gender $(\mathrm{O}=\mathrm{M}, \mathrm{l}=\mathrm{F})$ & 453 & 1065 & -89 & 970 & & \\
\hline Number of comorbidities & & & $1177^{* * *}$ & 16 & $815^{* * *}$ & 188 \\
\hline Mental disorders ${ }^{3}$ & 44 & 1153 & & & & \\
\hline Cardiovascular disease $^{3}$ & $2649^{*}$ & 1157 & & & & \\
\hline Genitourinary disease & 882 & 1003 & & & & \\
\hline Interferon beta $\mathrm{Rx}$ & $1329 * * *$ & 122 & $1419^{* * *}$ & 115 & $1392 * * *$ & 113 \\
\hline $\begin{array}{l}\text { Interaction of number of } \\
\text { comorbidities and number } \\
\text { of exacerbations }\end{array}$ & & & & & $310^{* *}$ & 92 \\
\hline $\mathrm{R}^{2}$ & 0.4265 & & 0.4959 & & 0.5131 & \\
\hline Adj $R^{2}$ & 0.41 .38 & & 0.4879 & & 0.5085 & \\
\hline F stat & $33.567^{* * *}$ & & $62.559 * * *$ & & $84.04^{* * *}$ & \\
\hline
\end{tabular}

${ }^{*} p<0.05,{ }^{* *} p<0.01,{ }^{* * *} p<0.0001$

Dependent variable $=$ total costs $1995-96$

'Number of exacerbations defined as hospitalizations or emergency room visits listing ICD-9-340 or 341, or prescriptions for oral or intravenous steroids; ${ }^{2}$ Age in years at beginning of study period; 3 Yes $=1$

included only control variables (not shown), while the second included the control variables plus the number of exacerbations (equations $1 \& 2$ ). This strategy was followed in order to determine if adding the number of exacerbations variable to the control variables significantly increased the predictive power of the regression equation. Because an increase in the coefficient of determination, or R2, that occurs upon the addition of variables to a regression equation may not necessarily indicate greater predictability when applied to the population, the form of the coefficient of determination adjusted for the degrees of freedom (adjusted R2) was used in the interpretation of the study results. Incremental $F$ ratios were calculated to determine whether a significant change in the adjusted R2 occurred with the addition of the variable indicating the number of disease exacerbations.

\section{Results}

\section{Descriptive Statistics}

Patients each experienced approximately one exacerbation during the study period as reflected in both the median and mean for this variable. The median patient age was 46 years old. The mean and median number of comorbidities was approximately five per patient. Patients had an average of 1.88 interferon beta claims filed in the two-year study period, with a median value of zero. Seventy percent of patients in this sample were female, which corresponds well with the epidemiology literature for the MS population. ${ }^{9,10}$ Twenty-three percent of patients had a claim indicating treatment for a mental disorder, $31 \%$ had a claim indicating cardiovascular treatment, and $47 \%$ had a claim indicating gentiourinary treatment.

Three hundred and twenty-four patients met the study inclusion criteria. Table 1 describes the total, pharmacy, MS-related pharmacy, medical, and MS-related medical costs to the MCO by the number of exacerbations experienced by the patient. Due to the small number of patients with more than three exacerbations, patients with three to eight exacerbations were grouped together in the descriptive statistics. All costs are presented in 1995-96 U.S. dollars. The majority of total costs were medical as opposed to pharmacy costs. 


\section{Regression Analysis}

Table 2 presents the results from the regression analysis. Significant predictors of total cost were the number of exacerbations, comorbidities, and interferon beta claims. Age and gender were not found to be significant predictors of total cost in this sample of patients. With regard to specific comorbidities, the presence of cardiovascular comorbidities was shown to be a significant predictor of cost. The continuous form of the variable representing the number of comorbidities was chosen for use in further analyses, because this variable proved to be a more significant predictor of total cost when compared to the three dichotomous comorbidity variables (mental, genitourinary, and cardiovascular).

Both the number of comorbidities and the number of exacerbations were found to be significant predictors of total cost in the regression equations. Because patients with comorbidities may require more extensive treatment for an MS exacerbation, this study hypothesized that a clinical interaction might exist between these two variables. In order to further explore relationship with total cost, another regression equation including the interaction of the number of comorbidities and the number of exacerbations was estimated. Equation 3 included only those variables found to be significant in all previous regressions-the number of comorbidities, exacerbations, and interferon beta claims, in addition to the interaction term between the number of comorbidities and exacerbations.

\section{Equation 3: TOTAL COST $=\beta_{0}+\beta_{1} E X C E R+\beta_{2}$ COMGRP + $\beta_{3} E X C E R{ }^{*} C O M G R P+\beta+1 B C L M S+\varepsilon 1$}

The adjusted R2 obtained in this regression indicated the variables accounted for approximately $51 \%$ of the variance in total cost. The increase in adjusted $\mathrm{R} 2$ that occurred upon the addition of the interaction term in equation 3 was significant (Finc $=11.35>$ Fcrit $=5.42$, alpha $=0.001$ ) when compared to an identical regression without the interaction term. Thus, when describing the relationship between the number of exacerbations and total cost, the number of comorbidities must be taken into account. The number of comorbidities and interferon beta claims remained significant in this regression equation $(p<0.0001)$. The interaction term between the number of comorbidities and exacerbations was also significant $(p<0.01)$. The main effect of the number of exacerbations was not significant in this equation, indicating that the predictive power of the exacerbations variable seen in previoús equations was most likely captured in the interaction term.

\section{Discussion}

The mean total cost to the MCO for the study sample $(n=324)$ of MS patients was $\$ 9,365 /$ patient/two-year period. Costs were highly variable with a standard deviation of $\$ 11,067 /$ patient/ two-year period. The principal determinants of cost were the number of exacerbations, comorbidities, and interferon beta claims. The presence specifically of cardiovascular comorbidities was also found to be a significant predictor of total cost. The relationship between total cost to the MCO and the number of

\begin{tabular}{|c|c}
\hline TABLE 3 & $\begin{array}{l}\text { Change in Cost Estimated for a } \\
\text { One-Unit Change in } \\
\text { Exacerbations, by Number of } \\
\text { Existing Comorbidities }\end{array}$ \\
\hline Number of comorbidities & Incremental change in total cost \\
\hline 0 & $\$ 359$ \\
\hline 1 & $\$ 669$ \\
\hline 2 & $\$ 979$ \\
\hline 3 & $\$ 1289$ \\
\hline 4 & $\$ 1599$ \\
\hline 5 & $\$ 1909$ \\
\hline 6 & $\$ 2219$ \\
\hline 7 & $\$ 2529$ \\
\hline 8 & $\$ 2839$ \\
\hline
\end{tabular}

The estimates presented in this table were obtained by rearranging Equation 3: Total Cost $=\beta_{0}+\beta_{1}$ Exacerbations $+\beta_{2}$ Number of Comorbidities $+\beta_{3}$ Interactions of numbers of exacerbations and number of comorbidities $+\beta_{4}$ Number of interferon beta $\mathrm{Rx}$ filled $+\varepsilon_{1}$ Equation 4 : change in cost per exacerbation $=\beta_{1}+\beta_{3}$ Number of comorbidities. Inserting the regression parameter estimates into this equation reveals that the increase in cost estimated for one additional exacerbation, in a patient with no comorbidities is: change in cos $/$ change in exacerbations $=359+310(0)=\$ 359$.

exacerbations a patient experienced was significant, and depended on the number of comorbidities.

The results of this study may be used to estimate the impact of a change in one of the independent variables on the cost of MS to a MCO. The effect of a change in the number of disease exacerbations on total cost will be used as an example. The interaction between the number of comorbidities and the number of exacerbations requires that both these variables be taken into account in estimating the impact of a change in one of these variables on the managed care budget. Equation 3 from Table 2 may be used to estimate the change in total cost that would be predicted with a change in the number of disease exacerbations experienced by the patient. This process is repeated for up to eight comorbidities shown in Table 3.

The ability to generalize the results obtained in this study is limited to the extent that the characteristics of the study population are similar to those of other populations. Although the prevalence of MS may be expected to be lower in Arizona than in the northern U.S., ${ }_{1}^{13}$ evidence suggests no differences exist among geographic locations in terms of disease severity or resource utilization. Furthermore, the prevalence of MS in this MCO sample_-100 out of 100,000 members-approximates the National Multiple Sclerosis Society's estimate that MS affects 95 out of 100,000 Americans. ${ }^{14}$ The generalizability of the estimated cost to the $\mathrm{MCO}$ is limited to the extent that coverage 


\section{Economic Assessment of the Relationship Between Disease Exacerbations and the Cost of Multiple Sclerosis}

and reimbursement rates in the MCO used in the study are similar to those of other managed care organizations.

The study inclusion criteria required patients to be continuously enrolled in the MCO for the entire study period. This enabled the study to estimate the per patient treatment cost during the two-year study period. However, the MCO undoubtedly incurred additional costs for those members who were not continuously enrolled during the study period. This study design would, therefore, result in a conservative estimate of the overall cost to the MCO of treating MS patients, because the costs incurred due to patients enrolled for only part of the study period would not be included.

Finally, the scope of this study is limited to the economic aspects of the use of disease-modifying agents in MS. The benefits of these agents in terms of quality of life, survival, and indirect costs are beyond the scope of this study but should be taken into account when making resource allocation decisions.

\section{Conclusion}

In the past, multiple sclerosis therapy has consisted of supportive care and symptomatic management. The introduction of disease-modifying agents for the treatment of MS has created the potential for this disease state to have a greater impact on managed care spending. This study provides a quantitative estimate of the cost of treating an MS disease exacerbation. It found that the number of exacerbations was a significant predictor of total cost to the MCO. Further, the study found that the total cost of treating an exacerbation depended on the number of existing comorbidities of the patient. Managed care decision makers may wish to target MS patients with multiple comorbidities for disease-modifying therapy, because the cost of an exacerbation was found to be higher for these patients. The methodological framework presented in this study may be used to assess the potential impact of a disease-modifying agent on a managed care budget.

\section{REFERENCES}

1. Anderson DW, Ellenberg JH, Leventhal CM, et al. Revised estimate of the prevalence of multiple sclerosis in the United States. Ann Neurol 1992; 31 333-36.

2. National Institute of Neurological Communicative Disorders and Stroke (NINDCS). Multiple sclerosis: A national survey. Washington DC: US Government Printing Office, 1985.

3. Jensen GA, Morrisey MA, Gaffney S, Liston DK. The new dominance of managed care: Insurance trends in the 1990s. Health Affairs. 1997; 16: $125-$ 36.

4. Frost, Sullivan. Changing patterns of drug reimbursement will bring big pay-offs to prepared companies. Media Release: 2 Feb 1998.

5. Zellmer WA. Comments of the American Society of Health-System Pharmacists: Pharmaceutical marketing and information exchange in managed care environments. Food and Drug Administration Public Hearing. Ocl. 19 , 1995

6. Inman RP. Disability indices, the economic costs of illness, and social insurance: the cost of multiple sclerosis. Acta Neurol Scan Suppl. 1984; 705: 46-55.

7. Bourdette DN, Prochazka AV, Mitchell W, et al. and the VA Multiple

Sclerosis Rehabilitation Study Group. Health care costs of veterans with multiple sclerosis: implications for the rehabilitation of MS. Arch Phys Med Rehabil. 1993; 74: 26-31.

8. Weiner HL, Hohol MJ, Khoury S], et al. Therapy for multiple sclerosis Neurol Clinics. 1995; 13: 173-96.

9. Lechtenberg R. Multiple Sclerosis Fact Book. Philadelphia, PA Davis. 1988. 10. Sadovnick AD, Ebers GC. Epidemiology of multiple sclerosis: A critical overview. Can J Neurol Sci. 1993; 20; 17-29

11. Fleming ST, Blake RL. Patterns of comorbidity in elderly patients with multiple sclerosis. J Clin Epidemiol. 1994; 47: 1127-32.

12. Greenberg PE, Stiglin LE, Finkelstein SN, Berndt ER. The economic burden of depression in 1990. J Clin Psychiatry. 1993; 54: 405-18.

13. Dalmady-Israel C. Multiple Sclerosis. Third Edition Pharmacotherapy, Chapter 54. 1997: 1167-77.

14. National Multiple Sclerosis Society. Memorandum No. 111-90. 